\title{
Hardware-in-the-Loop Testing in Racing Applications
}

Peter Waeltermann, Thomas Michalsky, Johannes Held dSPACE GmbH, Paderborn, Germany 


\title{
Hardware-in-the-Loop Testing in Racing Applications
}

\author{
Peter Waeltermann, Thomas Michalsky, Johannes Held \\ dSPACE GmbH, Paderborn, Germany
}

Copyright $\odot 2004$ SAE International

\begin{abstract}
Hardware-in-the-loop (HIL) simulation has established itself as a standard method of testing electronics components in the automotive industry. HIL simulators are now being used both by automotive suppliers and by automobile manufacturers. In racing particularly, the high cost of the vehicles and test benches means that frequent virtual test drives and tests are run on a simulator. The objectives are to ensure the greatest possible hardware and software quality before going over to the test vehicle or test bench, to cut costs, and to minimize the danger to test drivers.

Virtually all major formula 1 teams and some rally teams (WRC) use dSPACE simulators as HIL electronics test systems, for example, for the widely used electronics platform from Magneti Marelli and also for their in-house developments. The major focus is on ECU testing but aspects of ECU calibration will also be discussed. The paper describes this technology and presents solutions prepared for racing teams worldwide.
\end{abstract}

\section{INTRODUCTION}

Hardware-in-the-loop (HIL) simulation has become a standard method of testing electronics components in the automotive industry. HIL simulators are now being used by both automotive suppliers (for component tests) and automobile manufacturers (for network and integration tests).

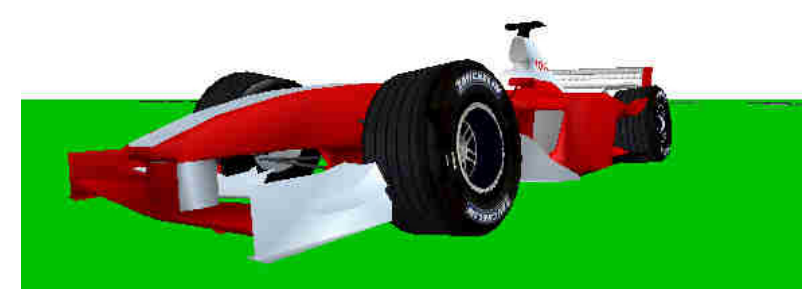

Figure 1: Virtual test drive with HIL simulation
In racing particularly, the high cost of the vehicles and test benches means that frequent virtual test drives and tests are run on a simulator. The objectives are to ensure the greatest possible hardware and software quality before going over to the test vehicle or test bench, to cut costs, and to minimize the danger to test drivers.

This paper presents applied hardware-in-the-loop simulation with reference to the particular requirements of motorsports (especially Formula One) and describes relevant customer solutions.

\section{HARDWARE-IN-THE-LOOP-SIMULATION}

In hardware-in-the-loop simulation, one or more real components (for example, electronic control units) are studied in interaction with components simulated in real time (models, for example, of the engine, transmission, powertrain and whole vehicle).

The interactions between real and simulated subsystems make tough real-time demands on these systems. High computing power (processor hardware for simulating the models, and the actuators and sensors) and $1 / O$ performance are therefore essential preconditions. Frequently, special hardware is used, for items such as fast engine signals and for generating and measuring CAN signals.

Using HIL simulation has the following advantages:

- The hardware and software of electronic control units (ECUs) can be tested (automatically) at an early stage because there is no need for a real engine or a real transmission to be present resulting in greater maturity before transfer to the test bench or vehicle.

- Fewer test drives and test bench experiments are needed, and also fewer vehicle prototypes or vehicle components - resulting in cost savings.

- Safety-critical components (such as drive-bywire) can be tested without danger. Component failures and associated emergency scenarios can be tested reproducibly.

- Difficult ambient conditions (winter tests, rain, high or low temperatures) can be achieved by simple parameter changes.

- Test bench experiments are highly reproducible. 
- The vehicle and its components can be visualized by $3-D$ animation. This functionality can be extended, right through to a real drive simulator for man-in-the-loop applications.

HIL simulation has established itself as a standard in the development process for passenger and sport-utility vehicles and is also growing in importance in motorsports.

\section{MOTORSPORTS REQUIREMENTS}

Clearly, many of the above advantages also apply to motorsports. Compared with the development of production vehicles, however, motorsports have their own specific and even tougher requirements:

- Test drives are several times as expensive in motorsports as for production vehicles, and moreover, they are partially restricted to only a few weeks a year (F1).

- When test drives are performed, all the relevant data has to be logged via the central electronics, as most racing cars cannot take additional measuring equipment on board. Moreover, it is not possible to sit in the passenger seat and modify ECU variables via a calibration system to fine-tune the system. This is only possible via telemetrics, if at all.

- Software development is characterized by weekly software releases and last-minute modifications (short iteration loops). The ECU parameters are individually adjusted for each racetrack.

\section{REQUIREMENTS WITH REGARD TO HIL SIMULATION:}

HIL simulation requires enormous processing power for the real-time simulation of the engine, powertrain, and vehicle dynamics components. Because of the dynamicity of the components and the high engine speeds, simulation step sizes of approx. $50-250$ us are usually used, as compared with production vehicle applications, where 500 us $-1 \mathrm{~ms}$ are more usual.

And engine speeds of up to $20,000 \mathrm{~min}^{-1}$, anglesynchronous generation of crankshaft and camshaft signals, and angle-synchronous capture of injection and ignition signals, require extremely fast $1 / O$ subsystems. Special hardware is generally used for this.

In motorsports, vehicles frequently have special sensors (LVDTs, thermocouples, linear lambda probes, etc.) and actuators (Moog valves, fast injection and ignition systems [1] that have only very limited use in production systems for cost reasons (for example, LVDTs for automated transmission). Mapping these for HIL simulation thus often requires special hardware or software solutions.

In contrast to production vehicles, the CAN busses in racing cars are always run at the highest baud rate (1Mbit/s). Typical tasks include capturing all the CAN messages of the ECU network and CAN restbus simulation for emulating absent network nodes. This is particularly important if the chassis and the engine are not developed on the same site (as is the case with most F1 teams).

\section{TYPICAL ECU ARCHITECTURES AND DETAILS}

The functionality of sensor systems in motorsports is hardly any different from that in production vehicles. However, there is a considerably greater volume of physical variables such as temperature, pressure, and air/fuel ratio to be captured. Racing cars generally have far more sensors $(>100)$. The sensors used are optimized for racing purposes (weight, dynamics, precision) and are often specially produced custom components.

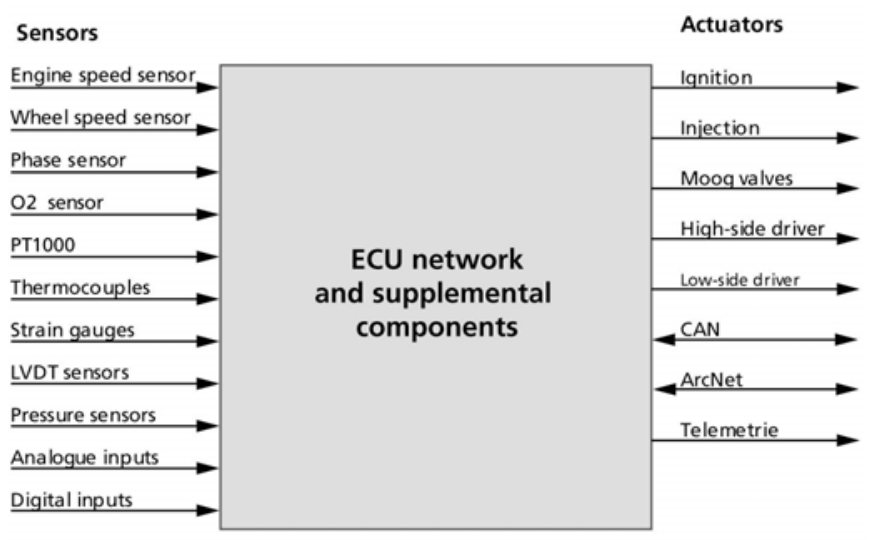

Figure 2: Typical sensors and actuators for racing applications

As vehicle electronics are increasingly decentralized, the ECU network is spread across almost the whole vehicle. With this decentralized structure, the number and weight of cable harnesses can be reduced. Fig. 3 shows a typical decentralized F1 vehicle topology with a few selected components.

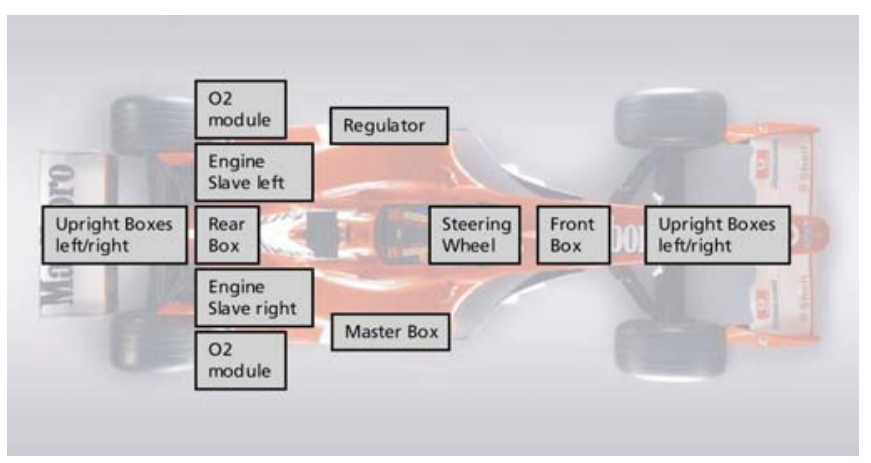

Figure 3: Typical decentralized F1 vehicle topology with selected components 


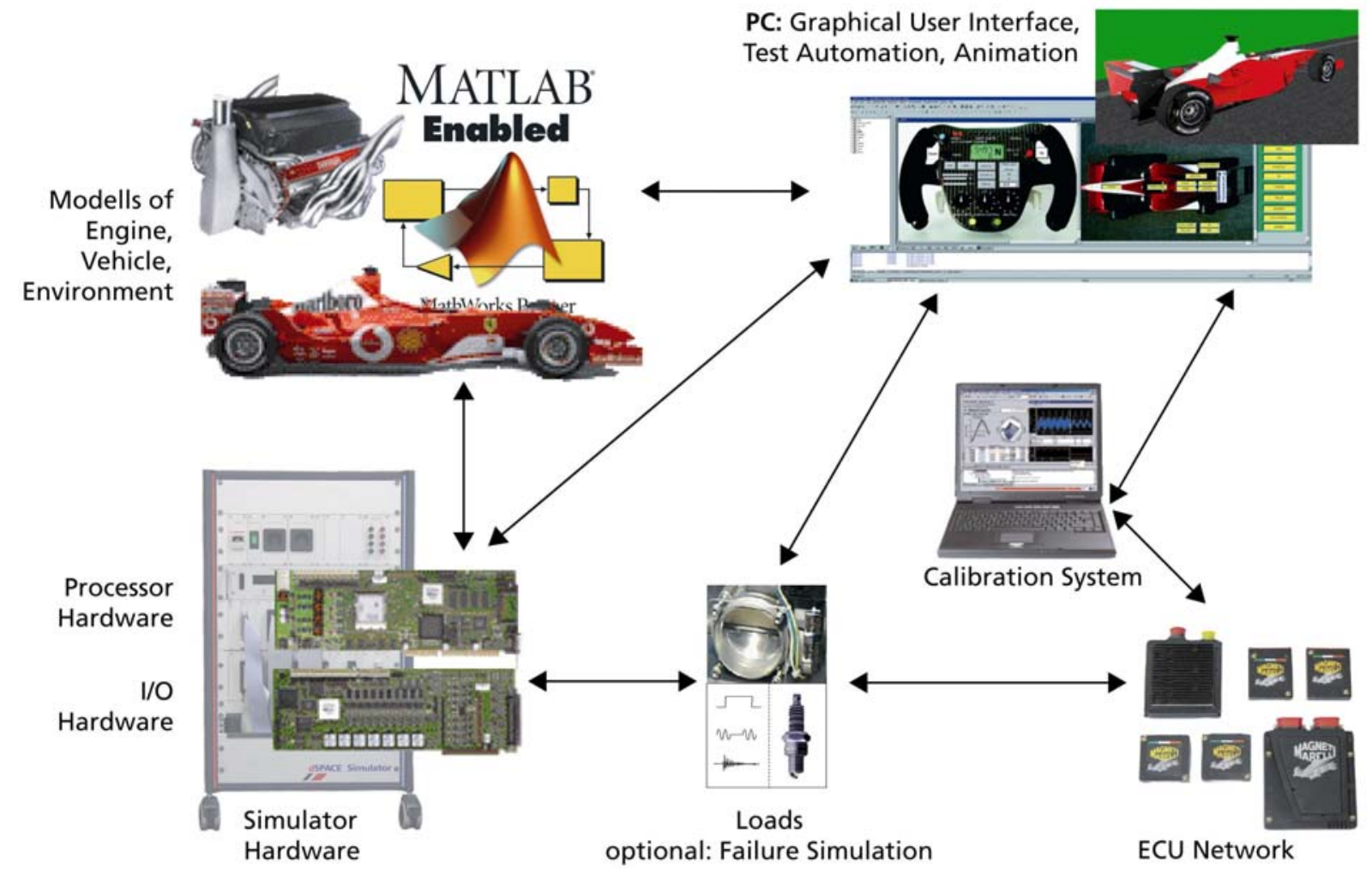

Figure 4: Components of a hardware-in-the loop test system for racing

\section{dSPACE SIMULATOR TECHNOLOGY}

Virtually all major F1 teams (e.g., Ferrari, Toyota [2]) and some rally teams (WRC) use simulators from dSPACE in Paderborn, Germany, for HIL electronic testing, for example, for the widely used electronics platform from Magneti Marelli (Step9, Step10, from 2005 also Step11), from TAG McLaren, and also for their in-house developments.

The simulators are used on the one hand for testing components, for example, the engine electronics subsystem. On the other hand, the entire system including all the essential elements of the ECU network is tested. The simulator has to be able to cope with the resulting complexity of the ECU network, and its hardware and software need to be scalable.

\section{OVERVIEW: THE COMPONENTS OF A SIMULATOR}

Fig. 4 shows the typical subcomponents of an HIL simulator and their interactions. Other important hardware components apart from the ECUs are the processor and I/O hardware, the signal conditioning, and the electrical failure simulation. On the software side, a graphical user interface (incl. animation environment) and dynamic models of the vehicle components are required. A test automation environment is also needed to automate test sequences. These components are described in detail in the following chapters.

\section{HARDWARE}

The core element is the generic real-time hardware, which comprises the processor board and $1 / O$ boards tailored to the actual application. The simulation requirements for testing the ECU and ECU's networks are therefore addressed by the solutions described below.

\section{Processor Hardware}

Dynamic models of the engine, transmission, powertrain, and vehicle dynamics are executed on a real-time processor hardware, such as the dSPACE DS1006 Processor Board at sampling rates of 50 us to $1 \mathrm{~ms}$. The board has an AMD Opteron $®$ processor with a clock rate of $2.2 \mathrm{GHz}$, allowing it to compute a mean-value engine model, a brake hydraulics model, and a vehicle dynamics model, including the entire $\mathrm{I} / \mathrm{O}$ for the engine and vehicle dynamics control, in less than 350 us. For even more complex models, or to connect several simulators, the boards can be networked to form distributed multiprocessor systems. 


\section{I/O Hardware}

The I/O capability of the simulators is directly derived from the actual application. Engine simulation, for instance, involves generating crankshaft, camshaft and knock signals synchronously to the engine angle, while injection and ignition signals are measured synchronously to the crank angle. Special hardware is generally used for this task, for example, the DS2211 HIL I/O Board, which is in widespread use in the automotive industry, for passenger cars and trucks as well as racing applications. The board provides the entire I/O for an 8-cylinder engine including signal conditioning, for example, and is cascadable to accommodate F1 10-cylinder engines either. Thanks to its modularity, further I/O boards can be added, covering all analog, digital and resistor channels. Regarding CAN nodes, the simulator hardware can provide as many CAN controllers as required.

\section{Mechanical set-up}

The mechanical simulator setup follows a generic approach, thus having a standard rack encompassing the individual components. Among others, the cabinet comprises real-time hardware, signal conditioning, failure simulation boards, load cards, break-out-boxes (BOB), etc.

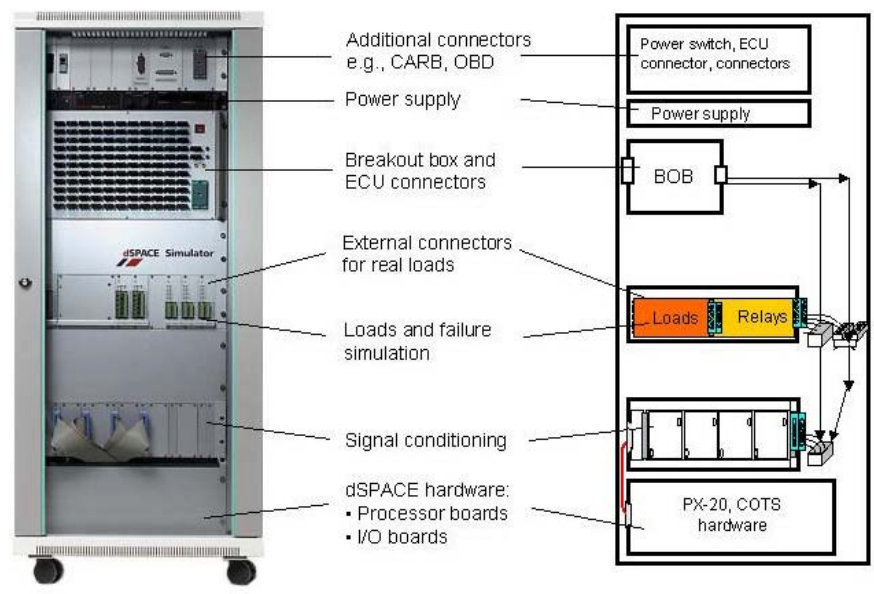

Figure 5: General structure of a dSPACE Simulator Full-Size

\section{Signal Conditioning}

Although the signal conditioning needed for many automobile-specific signals is provided by the DS2211 HIL I/O Board mentioned above, some signals still require special signal preparation, for example, for broadband lambda probes, LVDT sensors, thermocouples, etc.

\section{Electrical Failure Simulation}

As a rule, all manufacturers of racing ECUs specify electrically protected ECU pins, but the racing teams still perform validation against this specification. Electrical failure simulation is an integral component of the simulator, and allows actions such as switching ECU pins against $\mathrm{V}_{\mathrm{BAT}}$ or ground and interrupting lines.

\section{SOFTWARE}

The software for the test systems basically consists of two components, the software for model and $1 / 0$ definition and the user control software for the test system.

\section{Software for Model and I/O Definition}

With the dSPACE test systems, modeling and $1 / O$ specification are based on MATLAB ${ }^{\circledR} /$ Simulink $^{\circledR}$, the quasi standard for describing dynamic systems in block form. For defining the dynamic behavior of engine, transmission, and vehicle dynamics, Simulink and Stateflow ${ }^{\circledR}$ have established their market position.

Extensive Simulink block libraries are available for defining $\mathrm{I} / \mathrm{O}$ in the same Simulink environment. dSPACE's Real-Time Interface (RTI) is a Simulink block library that not only allows analog and digital inputs and outputs to be specified in Simulink. It also contains easyto-use blocks for the most complex situations (see Fig.6).

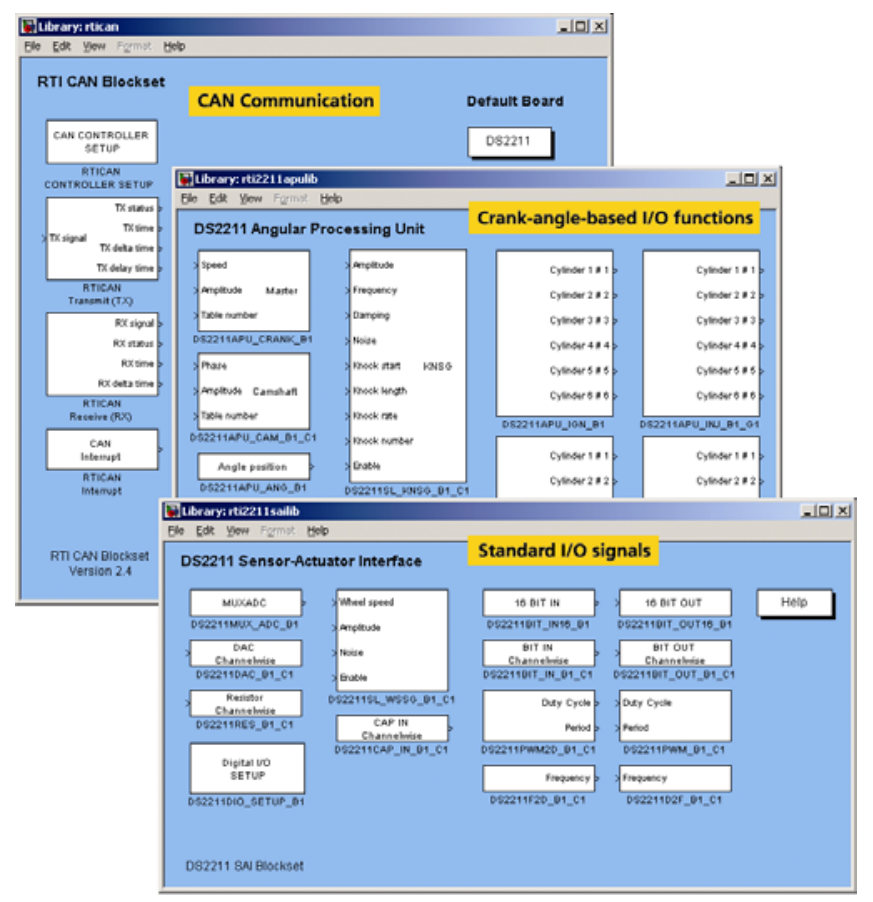

Figure 6: Simulink block library for I/O signal specification 
One example is the angular processing unit (APU) of the DS2211 HIL I/O Board. This intelligent I/O subsystem performs all crankshaft angle based functions. The APU receives the current engine speed from the real-time simulation and integrates it with the current crankshaft angle, at an update rate of $4 \mathrm{MHz}$ and a precision of $0.01^{\circ}$. Digital or analog crankshaft and camshaft signals, and knock signals, are then generated at the same precision. Angle-synchronous capture of injection and ignition signals (incl. multiple injection and ignition) is based on the APU, with the stated time and angle resolution.

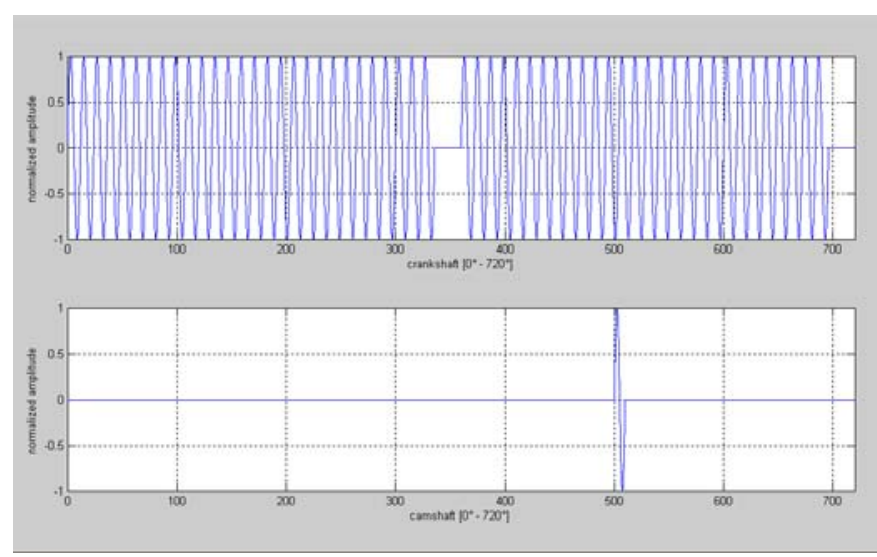

Figure 7: Crankshaft and Camshaft signal of a typical racing application (MATLAB ${ }^{\circledR}$ plot)

Although these hardware-related functions are processed on an FPGA, the ignition sequence, and the crankshaft and camshaft signals, are completely specified in Simulink, by means of wave tables (MAT files).
The signals can either be generated synthetically (e.g., sinus function for variable reluctance sensors) or come from actual measurement. It is also possible to switch between different wave tables at run time, for example, to simulate a faulty toothed wheel. With analog sensors, the amplitude of the signal can also be adjusted to engine speed behavior $\left(U_{\text {amplitude }}=f(r p m)\right)$. This is particularly important for racing systems from Magneti Marelli.

For defining CAN messages to read from or write to the CAN bus, RTI also provides block libraries that allow CAN data (DBC format) to be read in so that messages from the test system can be coded and decoded with the correct signal scaling.

In addition to the component models, ECU models are also frequently implemented ("soft ECUs") or are already available from function development. The soft ECUs are used in offline simulation or if real ECU prototypes are not yet available. Central switches in the I/O model can be used to switch back and forth between the simulated and the real ECU. The soft ECUs can also be employed for dynamic CAN restbus simulation, making it possible to mix real and simulated CAN network nodes, for example, if not all CAN nodes are available in real form.

As already mentioned, because of the high clock rates and associated processing power requirement, multiprocessor (MP) systems are often used. Here too, it is important to specify the entire MP system, including interprocessor communication, from one Simulink model. RTI blocks with appropriate sensor and actuator scaling, and dynamic model behavior, therefore allow seamless specification based on one MATLAB ${ }^{\circledR} /$ Simulink $^{\circledR}$ model. The MATLAB ${ }^{\circledR}$ Real-Time-Workshop (RTW) is then used to generate the $\mathrm{C}$ code for the real-time hardware from this model. Thus, it is generally not necessary to have a knowledge of $\mathrm{C}$ programming to generate the simulator code.

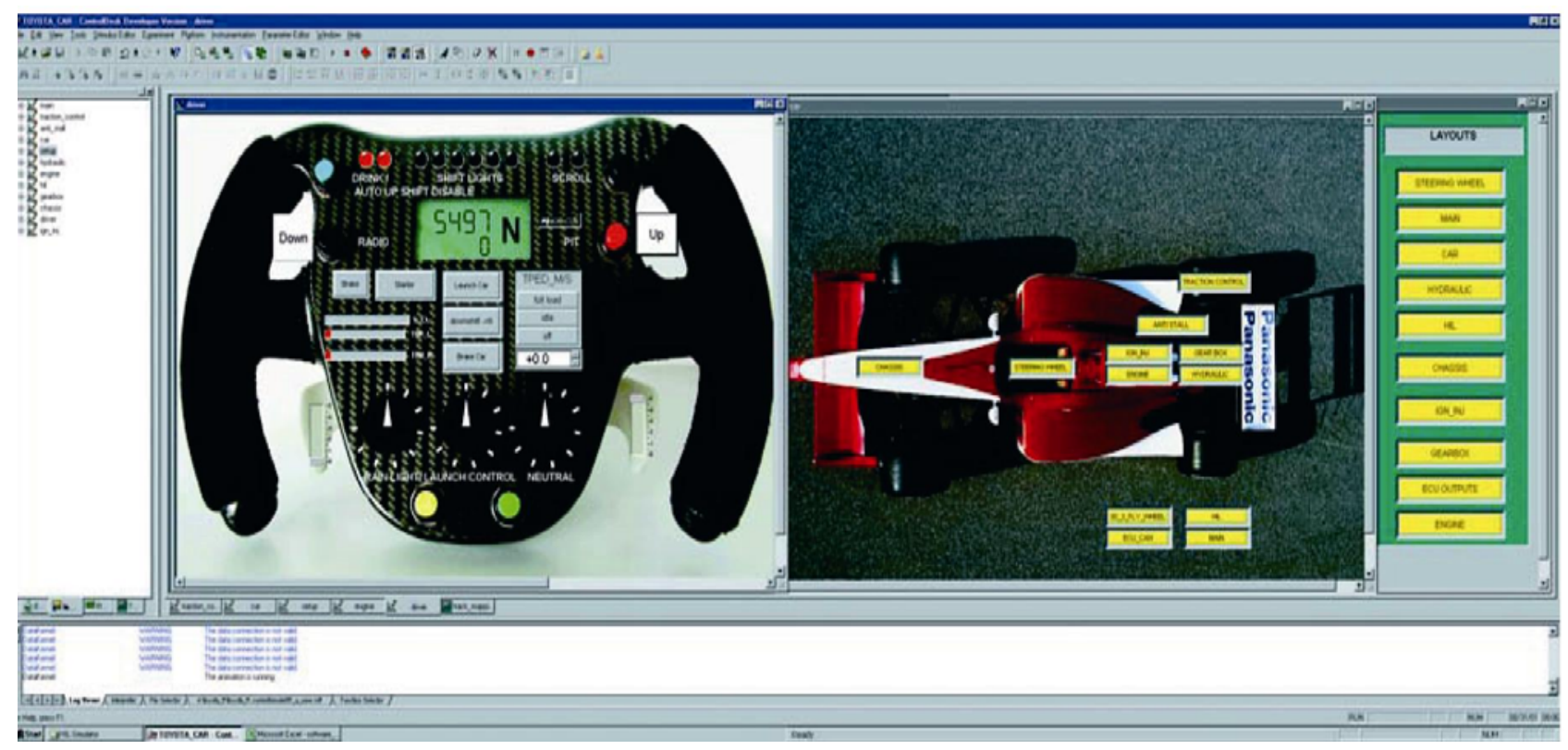

Figure 8: Graphical User Interface based on ControlDesk Layouts [2] 


\section{Interactive Operation}

The ControlDesk program is used for interactive operation of the test system. ControlDesk allows users to generate convenient, graphical user interfaces (layouts) with a great variety of control elements, from simple GUI elements (push-buttons, displays, radio buttons, etc.) to complex plotters and photorealistic graphics. Fig. 8 shows examples of application-specific layouts.

In addition, ControlDesk is used to control the simulation and electrical failure simulation, and to manage interactive experiments. A macro recorder and programmable switch elements for convenient maneuvering between layouts round off the functionality. Apart from this interactive work on the simulator using ControlDesk, other important aspects of working with the $\mathrm{HIL}$ test system are automated testing and (for vehicle dynamics particularly) the animation of motion data.

\section{Real-Time Models}

To map the real world in the simulator, real-time models for all the relevant vehicle components are implemented. For example, the engine, transmission, powertrain, and engine dynamics (incl. wheels) are modeled and then computed on the real-time hardware. The models are generally provided by the simulator customers, but sometimes commercial models are integrated, such as ve-DYNA/en-DYNA [3] from TESIS, Munich, Germany, and CarSim [4] from MSC (Mechanical Simulation Corporation), Ann Arbor, MI, USA.

The dynamics of the engine, transmission and powertrain must be represented precisely in order to test dynamic control behavior. For example, if the gearshift strategy needs to be checked or optimized, the following items are investigated:

- Environment model (track, ground surface, irregularities, etc.)

- Vehicle model, incl. aerodynamics (ground effect) to determine wheel forces

- Tire model (to compute slip)

- Powertrain model (for torque distribution in the differential)

- Transmission model (gearshift dynamics and clutch)

- Engine model (engine torque and speed computed from injection and ignition during gearshift)

These are all tested in interaction with the real ECUs.

When one considers that the entire gearshift operation usually takes only between $20 \mathrm{~ms}$ and $40 \mathrm{~ms}$, it is obvious that the quality of both the model and the simulator hardware has to fulfill very tough requirements.
There are generally two approaches to engine models:

a) Extended mean value models in which the whole dynamic engine process is regarded as a continuous energy flow [5] Sampling rates of 250 - 500 us are usually sufficient to simulate Formula One engines with this approach.

b) Models based on the Vibé curve, in which each cylinder-selective combustion process (engine torque behavior) is described separately and computed on the basis of individual injection and ignition signals, and of the intake manifold pressures. For common Formula One engine speed sampling rates, between 50 and 100 us are needed to describe the engine torque behavior precise enough for ECU testing.

\section{Test Motivation in Racing}

The requirements for the test scenario are in large part the same in racing as for testing production vehicle ECUs. However, there are some specific aspects to be taken into consideration.

\section{Component Testing}

The ECU software is constantly being further developed and modified. There are new releases every week, and these have to be tested before they can be put in the racing vehicle. Component testing also includes tests on submodules of an ECU network before they are integrated into the overall system.

Network Testing

Network tests are derived directly from the component tests. Sufficient validation is required that the individual ECU network components add up to a functioning overall system, i.e., whether communication is via CAN busses or ArcNet functions.

\section{Regression Testing}

Any time one element in the ECU network is modified, all functions have to be tested again to ensure that complete functionality has been preserved.

\section{Limp Home Testing}

The ECUs must guarantee that even if sensors and actuators fail, the overall system will still go on working. Suitable limp-home strategies allow the racing car to continue moving, as much as possible, despite functional faults. And before safety-relevant functions can be integrated into the vehicle, the possibility of function failure has to be excluded under all circumstances. This type of test includes investigating whether drive-by-wire systems react to the failure of specific sensors (e.g., the throttle valve sensor) in the manner expected.

\section{$\underline{\text { Test Automation }}$}

Further development of the extremely complex ECU functionality, and very short times between releases, make it essential to automate tests. Automation also makes it easy to implement $24 / 7$ operation, which can dramatically shorten development times. The racing teams implement tests in different ways. The following methods are used (Fig. 9): 


\section{ControlDesk Stimulus Editor}

Graphical description of the test sequence using the signal patterns, that determine, for example, the behavior of a sensor. The Stimulus Editor provides access to models of all sizes. It is also possible to specify the control flow (e.g., If-Then-Else) within the test sequence. Textual Description

An integrated script language is used to describe the test procedure.

\section{Test Description in Simulink}

The test sequences are implemented in the Simulink model. Items such as an ECU input are stimulated by a test signal via standard Simulink blocks.

\section{AutomationDesk}

Tests must be systematically automated, appropriately structured, and reused, in order to achieve the targets regarding test depth and test width, and therefore also quality. In the recent past, test automation applications were frequently developed in programming languages and were customer-specific. Unlike previous solutions, AutomationDesk allows tests to be constructed in graphical form, without knowledge in programming. Further advantages include integrated test management, a powerful mechanism for creating custom test libraries, result management and integrated report generation (Fig. 10).

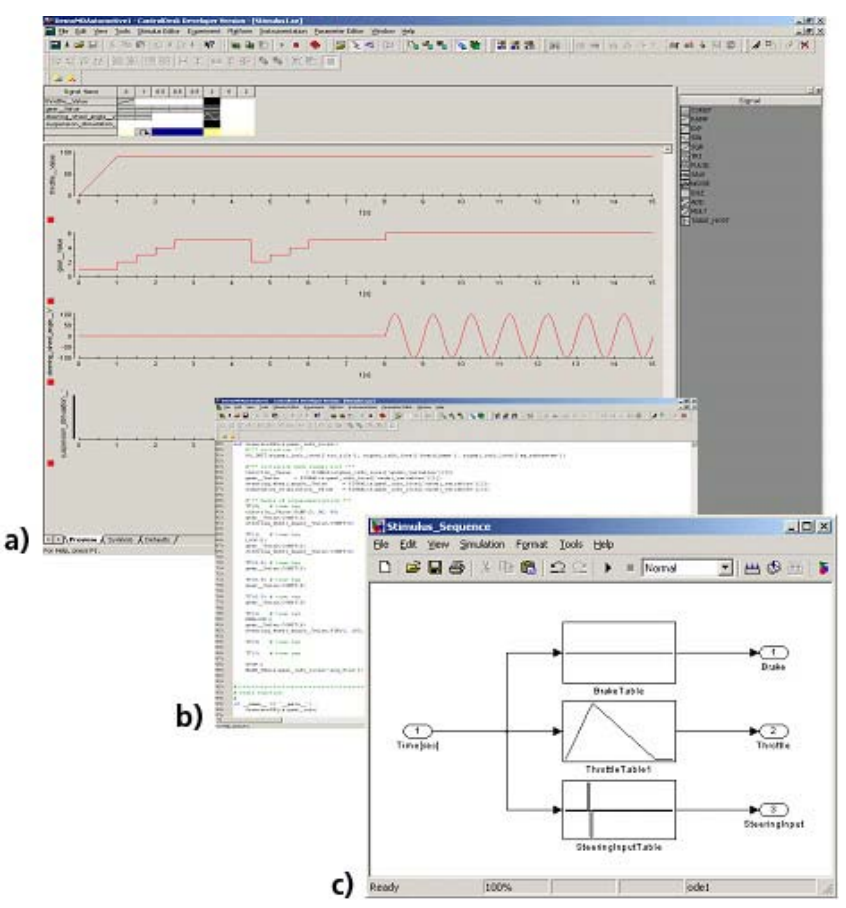

Figure 9: Different approaches to defining ECU tests: a) ControlDesk Stimulus Editor, b) Scripting, c) Test definition in the model

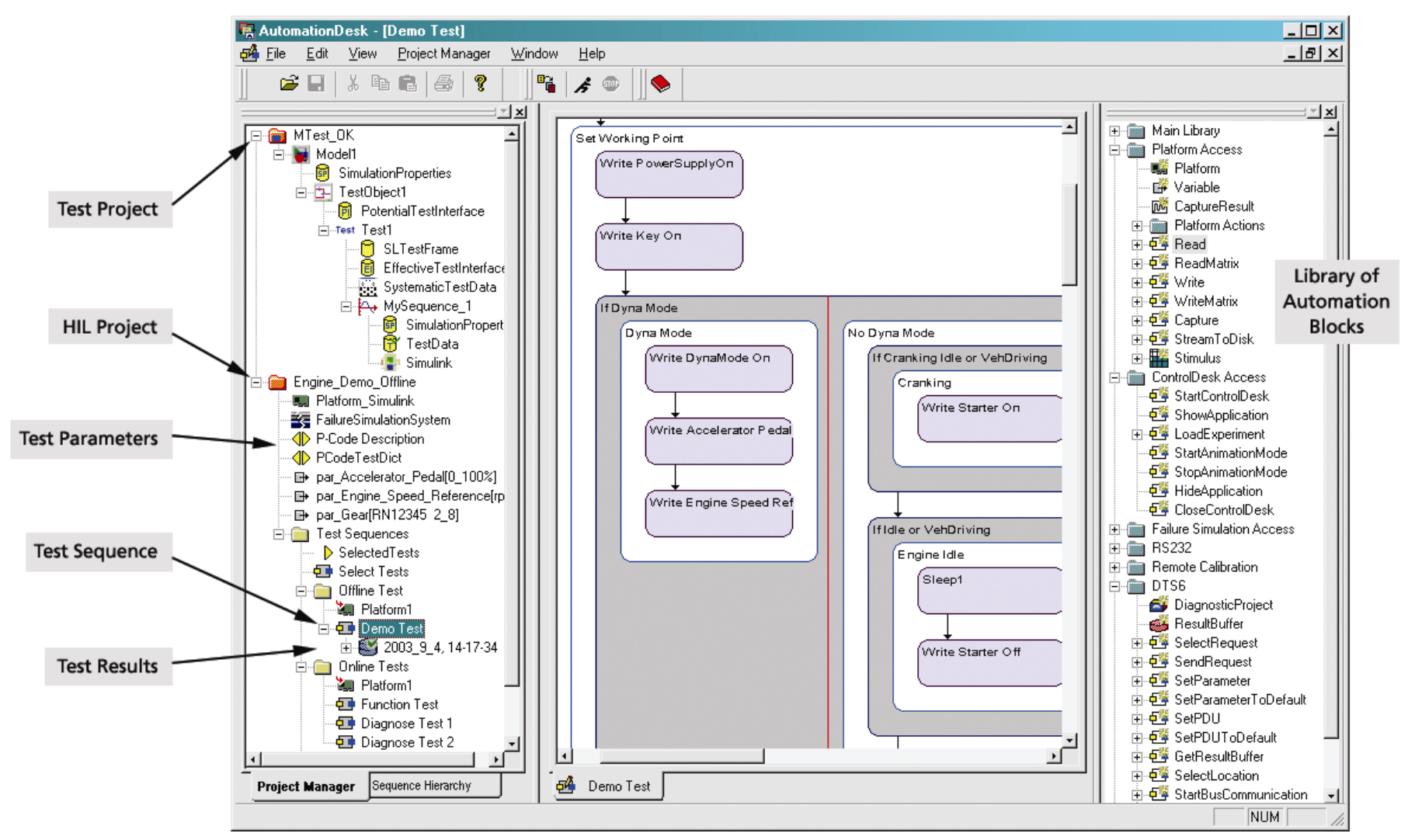

Figure 10: AutomationDesk, an integrated environment for automated testing [6] 


\section{APPLICATIONS}

\section{SYSTEM DESIGN IN RACING}

There is no such thing as "the" standard racing simulator. For component testing, the functional scope of the simulator, and therefore also the I/O capacity, can be much smaller. For example, a component testing system (Fig. 11) can do without items such as ignition and injection channels if tests are to be performed on the transmission or powertrain.

Simulators for motorsports are precisely tailored to the ECUs to be tested. Their size depends on factors such as the following:

- The number of ECU pins

- Whether an ECU network is involved (e.g., Magneti Marelli Step10/11, McLaren MCU 300/TAG-300) or a single ECU (e.g., Magneti Marelli Step9)

- How many additional components have to be integrated into the overall setup

- The scope of the tests (component tests, system tests, etc.)

- The processing requirement of the model, i.e., whether a multiprocessor system is needed

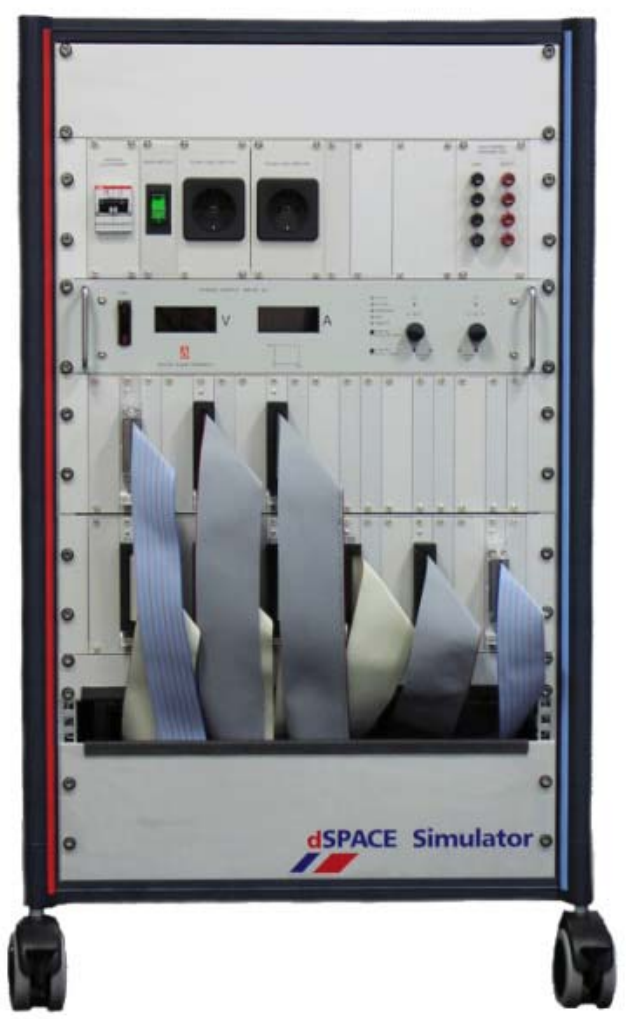

Figure 11: Simulator for ECU component testing

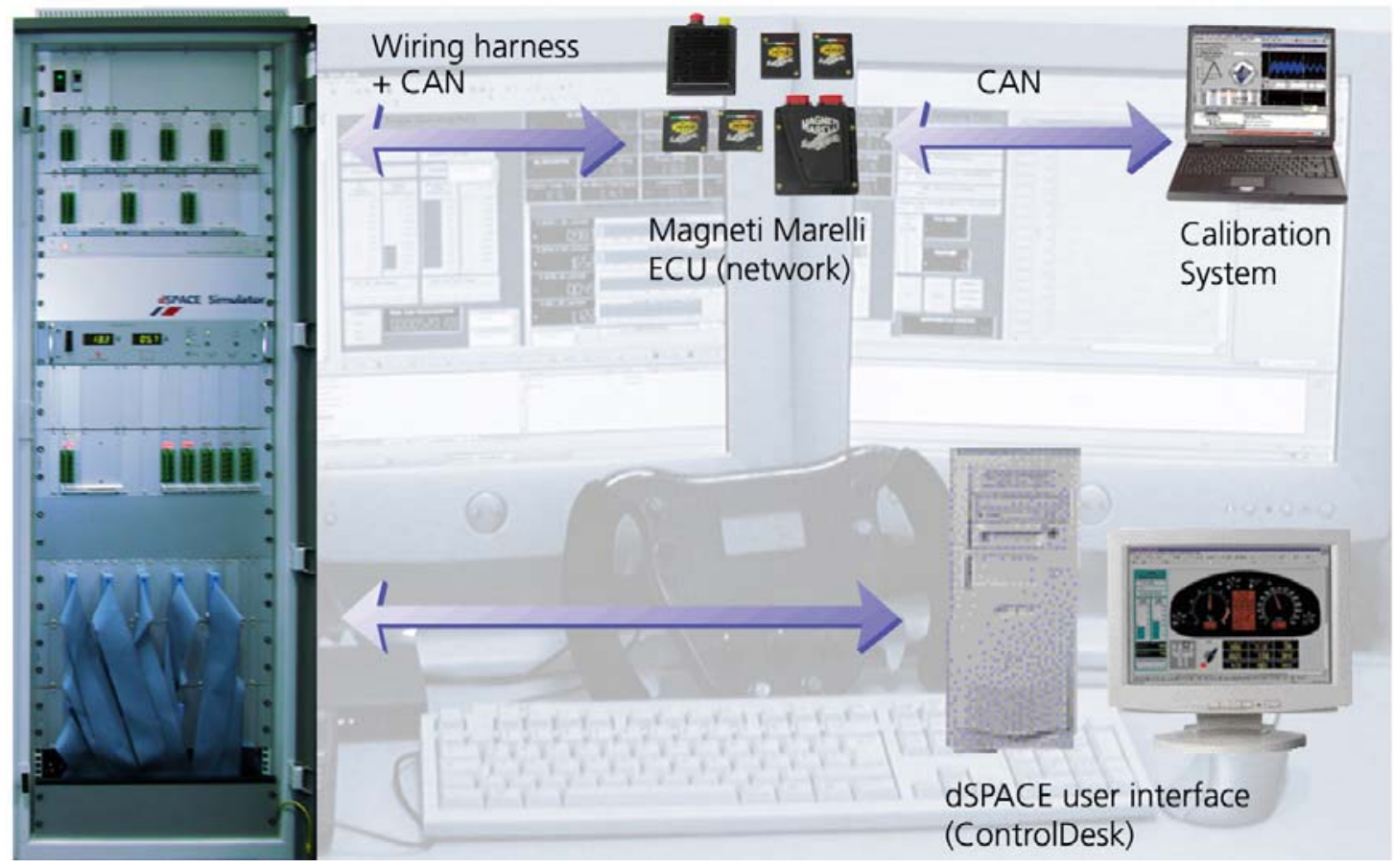

Figure 12: Laboratory setup of the hardware-in-the-loop system [2, 7] 


\section{SENSORS AND ACTUATORS}

The sensors and actuators are implemented in off-theshelf signal conditioning modules for racing simulators The simulator hardware is precisely tailored to the scaling in the Simulink model. In contrast to productionvehicle components, sensor characteristics in racing applications are captured individually. The $\mathrm{I} / \mathrm{O}$ model is parameterized via a start-up script. The racing teams generate the major part of the Simulink model automatically, so that they can perform actions such as integrating new sensors into the simulation in a matter of seconds.

These are examples of components installed in motorsports simulators (by analogy to the components described in chapter dSPACE Simulator Technology):

\section{Linear Lambda Probe}

Broadband lambda probes are mostly used in motorsports to operate the racing engines at optimum performance (lambda appr. 0.9). The simulator is equipped with a lambda probe module that sets the pump current according to the lambda value provided by the model and if necessary also varies the internal resistance of the Nernst cell with reference to the exhaust temperature. The characteristics are parameterized directly in Simulink, for example, by inserting the 1-D look-up I $=f($ lambda). In an ideal case, the manufacturer's specification or the developers' own measurement series can be transferred to the model for this.

\section{LVDT Sensor}

To detect the positions of the clutch or to capture the brake disk wear, racing vehicle developers frequently use (linear variable differential transformer (LVDT) sensors. To simulate the sensor, the LVDT module in the simulator reads the excitation signal (primary side) and makes the output signal (one or two secondary coils) available to the ECU in the correct phases. The deflection is placed directly in the model as the function $\mathrm{U}_{\mathrm{DAC}}=\mathrm{f}(\mathrm{s})$.

\section{Active Wheel Speed Sensor}

Active wheel speed sensors generally have a current interface whose level is provided by the simulator. The frequency to be output is scaled in Simulink according to the sensor data (teeth/wheel circumference) and converted, for example into a 7/14 mA level.

\section{Moog Valves}

The current-controlled Moog valves are read in indirectly via voltage measurement at a shunt. In the model, the voltage is converted into control current, which in turn is the input for the dynamic Moog model. Usually, the effective deflection of the actuator is computed via the integration of the coil current (which corresponds to a specific control speed). Here too, the transfer behavior from coil current to control speed is included in the model as a look-up table.

\section{Thermocouples}

Thermocouples have a very low output voltage, which is provided with great precision by a special $\mathrm{mV}$ module. The control range, e.g., $0-50 \mathrm{mV}$, is specified in the model.

\section{MODEL STRUCTURE}

To fulfill motorsports requirements with regard to processing time, the simulators are mainly multiprocessor systems. Typically, the model is split into the I/O part and the dynamic model, each of which has its own processor board.

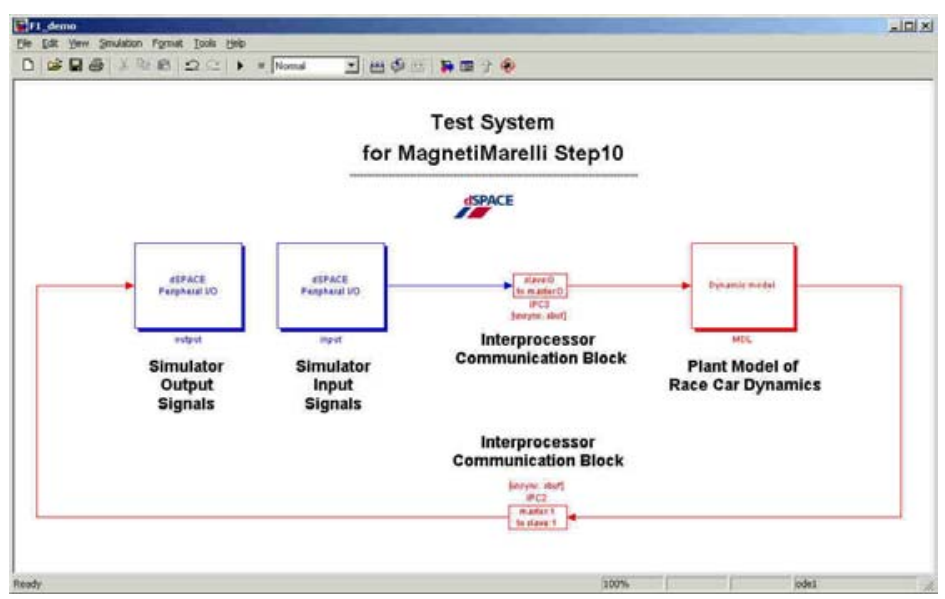

\section{Figure 13: Simulink model structure for a multi- processor system incl. interprocessor communication}

\section{IGNITION AND INJECTION}

The DS2211 always provides angle-based ignition and injection pulse capture and flags the absence of pulses. It is extremely important to ensure that any cut-off is detected, because when the ECU cuts off the ignition or injection, the associated status information must be generated as soon as possible in the simulator. The pulse status for each cylinder is then used within the model, to reflect the torque drop. When there is no pulse, the DS2211 generates a pulse state of 0 . Typically this information is included in the computation of the engine torque. (Fig. 14)

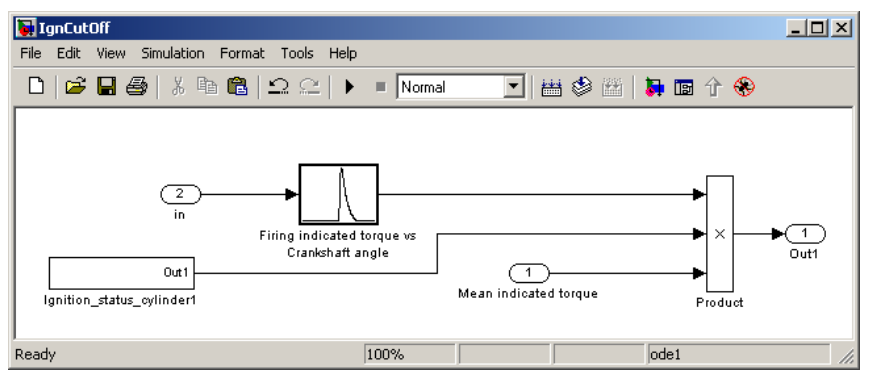

Figure 14: The torque computation must take ignition cut-off into account 
The resolution of the engine variables depends directly on the sampling rate at which the Simulink model is computed. For the best possible resolution, for example, of the pressure behavior in the cylinder, the sampling rate must be as low as possible.

An example: Revolutions of $18000 \mathrm{rpm}$ correspond to a change speed of the crankshaft angle of $108 \% \mathrm{~ms}$ or $0.108 \%$ us. If the dynamic model is computed at $1 \mathrm{kHz}$, the resolution is only $108^{\circ}$. A crankshaft angle of $21.6^{\circ}$ can be achieved at $5 \mathrm{kHz}$, and of $5.4^{\circ}$ at $20 \mathrm{kHz}$. Obviously, the real-time hardware must compute the model as fast as possible to achieve optimum resolution. Especially for engine models that compute the combustion behavior in the cylinder (Vibé curves), model clock rates of 50 us are desirable (Fig. 15).

\section{REAL-TIME PROFILING}

In all cases, the application as implemented must include the model's behavior over time. In addition to the specified sampling rate, the effective computing time required for each task is also represented. If a violation of real time is detected, it may be necessary to increase the sampling rate. The timing is profiled with the help of ControlDesk, giving access to vital simulation time data such as sample rate, task turn-around time, task call counts, task overruns, etc. It is crucial to check the application's behavior over time, i.e., how much headroom actually is available taking into account time consumption peaks.

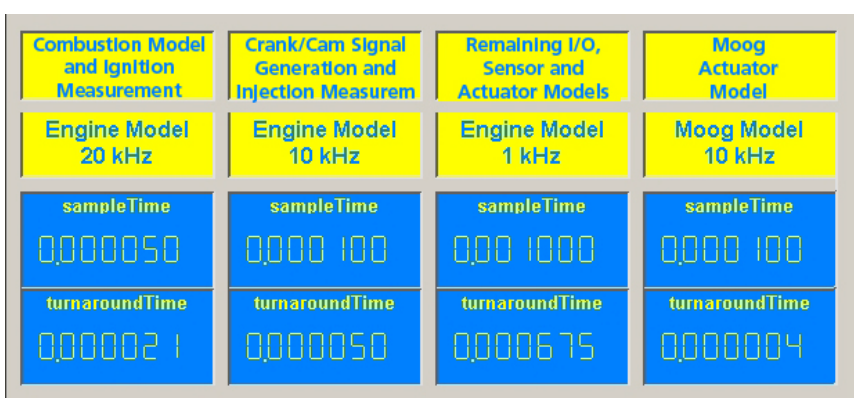

Figure 15: ControlDesk Layout showing the effective computing time in seconds for individual simulator tasks by means of numeric displays

\section{REAL PARTS}

There are many different reasons for integrated real parts into the simulator. For example, if there are submodels that are not (or not yet) available in Simulink, the control loop concerned can still be closed by including the real part. Some components can be very difficult to model, so engineers fall back on integrating the real parts, for example, a real throttle valve, to close the control loop for simulation.

With racing car components that are controlled by the driver, such as the steering wheel, pedals, or console (rally cars), the aim is to make the simulation as realistic as possible.

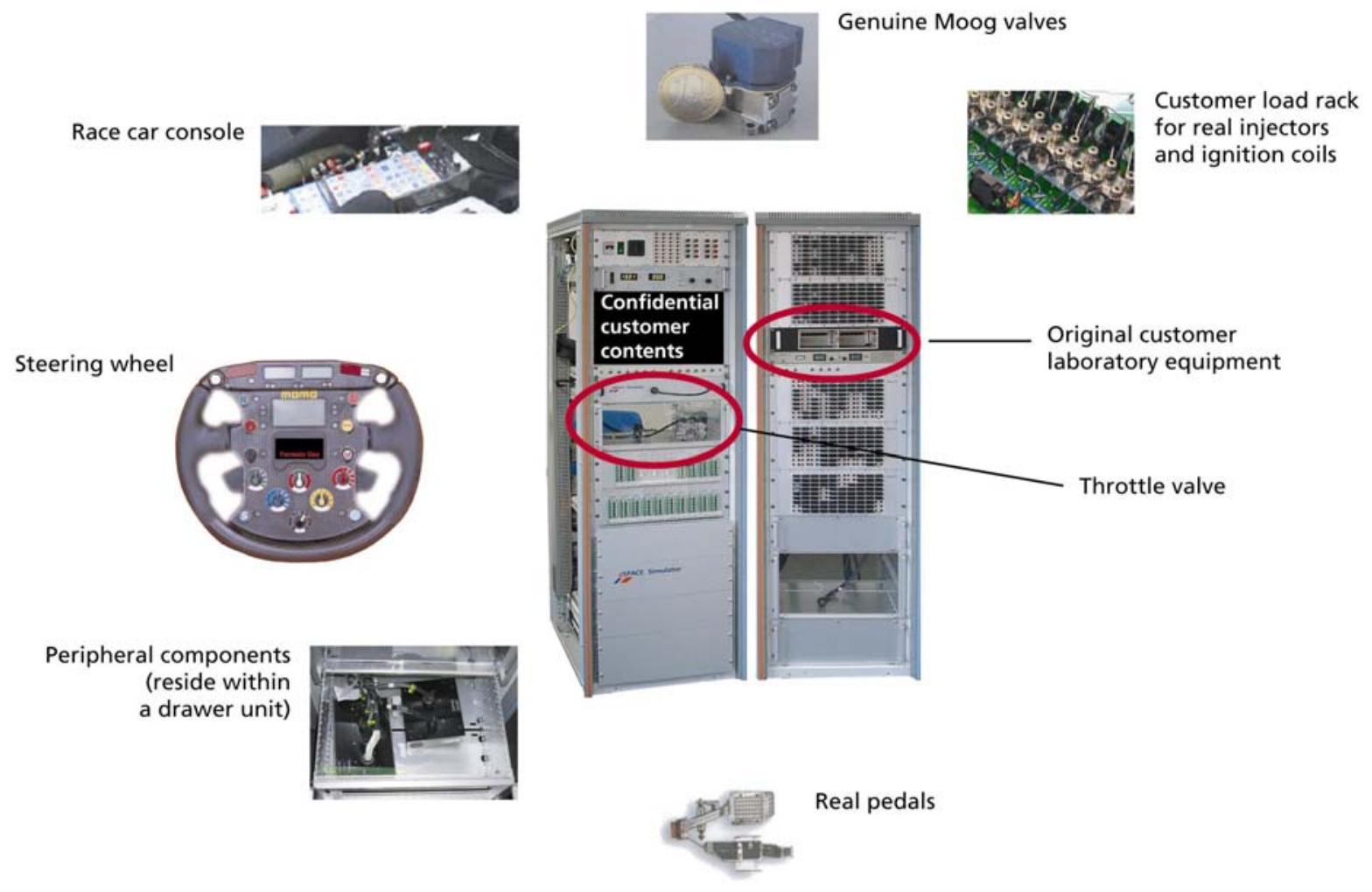

Figure 16: Simulator for rally (WRC) applications with original customer components 


\section{Junction Boxes}

The connections in racing vehicles are generally military industrial cylindrical connectors (MIL). To connect the ECU network to the simulator, specific junction boxes are used. As a rule, the boxes are made by the teams, and they can be located inside or outside the simulator. This makes it possible to use the original vehicle cable harness, or parts of it, in the simulation environment.

\section{GATEWAY OPERATION}

The simulator is able to corrupt specific signals that are exchanged between the ECUs and other peripherals in the vehicle. This method of intervention (gateway) is based on the principle of the simulator fetching all the relevant signals, corrupting them in real time, and outputting them to their recipients. It does not matter whether the gateway is for discrete signals or for CAN bus signals.

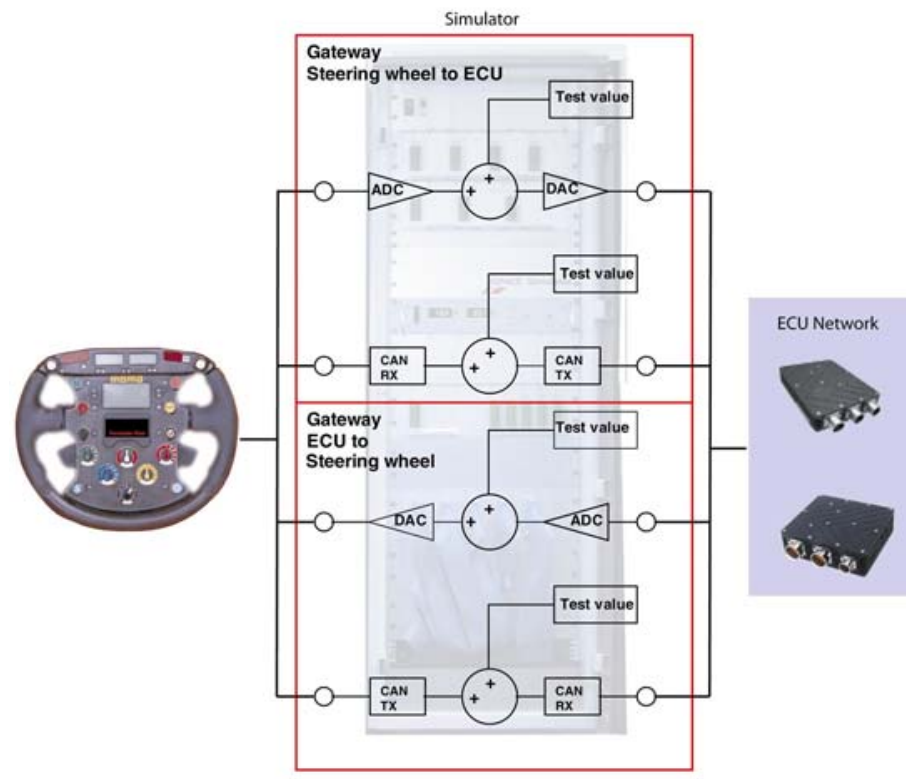

\section{Figure 17: F1 steering wheel as an example of gateway operation}

For the steering wheel, for example, analog signals from position sensors are read in on ADC channels. These signals can then be output again unchanged (correct behavior), or their values can be corrupted by a test value on the operator's PC and then sent to the ECU via the appropriate analog output port (incorrect behavior). CAN data are also manipulated on the same principle. Signals received from both the ECU and the steering wheel are available as physical values according to CAN scaling (offset and gain). These values can be corrupted on the basis of physical units and then put on the bus by the simulator. Besides changes to individual CAN signals (such as checksums), entire messages (missing, wrong timing), and even the complete absence of a bus node can be simulated and their effect on the rest of the network investigated (see [8]).

\section{USER INTERFACES}

Specific ECU functions have their own control layouts, in addition to the general layouts for system control (such as voltage supply). For example, different layouts are available for low-level functionalities such as ignition and injection and higher-level control functions such as launch control and traction control.

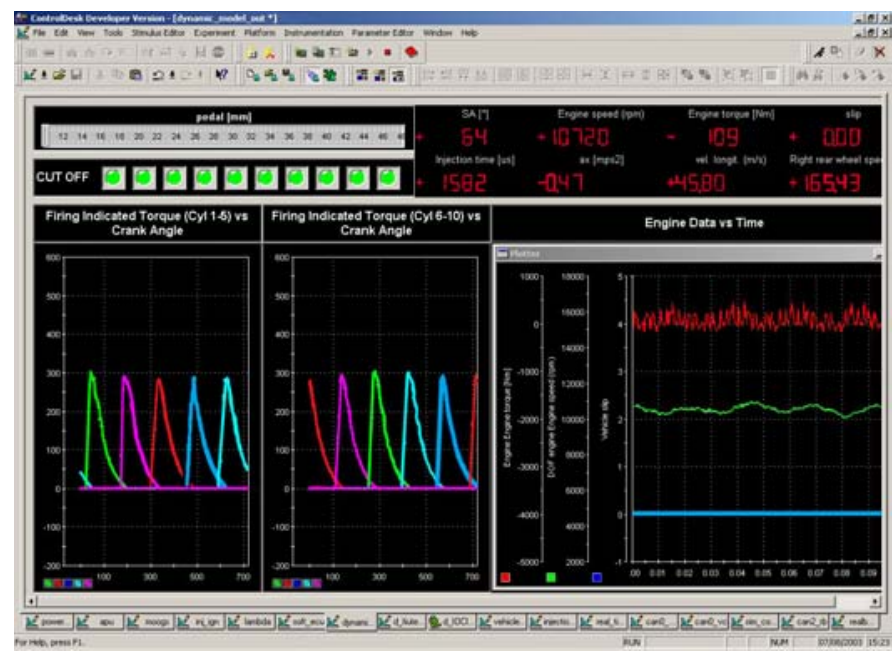

Figure 18: F1 simulator layout for dynamic model data analysis

For pure channel stimulation, stimulus layouts are also used. When a test value has been set in a layout, its correct implementation in the ECU can immediately be checked by a calibration tool. Fig. 19 shows a test layout from a rally application, in which all speed signals can be specified on one layout. These specifications can of course also be completely automated.

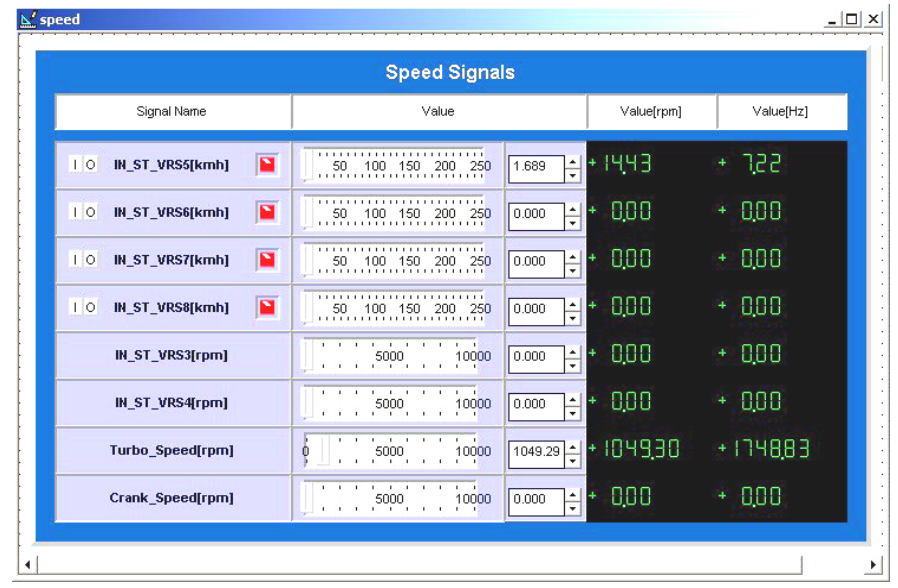

Figure 19: Test layout in a rally application for stimulating individual channels 


\section{AUTOMATED STIMULUS TESTS}

The ECU is stimulated by the precise data recorded during an actual test drive or in test bench trials. Feeding this data into the simulator brings the ECU to the operating point near which a function fault, for example, was detected, making it possible to investigate in detail in the laboratory.

If a Simulink model of the ECU functionality is available, function tests that validate the software ECU against the real hardware can also be automated.

\section{ANIMATION}

Animating the vehicle is done with dSPACE MotionDesk; a tool, which provides 3-D animation of mechanical system in a virtual world. There are essentially three steps involved in animation for HIL simulation:

- The motion data of the dynamic vehicle model (roll/pitch/yaw, translation) must be linked to the bodies to be animated (chassis, wheels, steering wheel, etc.) via special Simulink blocks (MotionDesk library and others) to create kinematic chains.

- Geometries from the CAD systems of racing teams are very complex and have a large number of polygons (>50000). These large polygon counts cannot be handled under realtime conditions on a PC. Thus the original data formats of these CAD systems must be transferred to a data format (VRML2) that is suited to animation.
- $\quad$ The vehicle must be embedded in a virtual, 3-D landscape. For motorsports applications, the animation scene is given items such as a track definition that corresponds to real racetracks or the team's real test track.

When these steps have been completed, the vehicle dynamics can be analyzed in the virtual 3-D world in MotionDesk in parallel to the simulation run. As a rule, real steering wheels are available on the simulator to allow man-in-the-loop operation, i.e., the user can steer the simulated vehicle, interacting with the ECU network while at the same time receiving visual and acoustic feedback (e.g., by sound generation to mimic the engine speed).

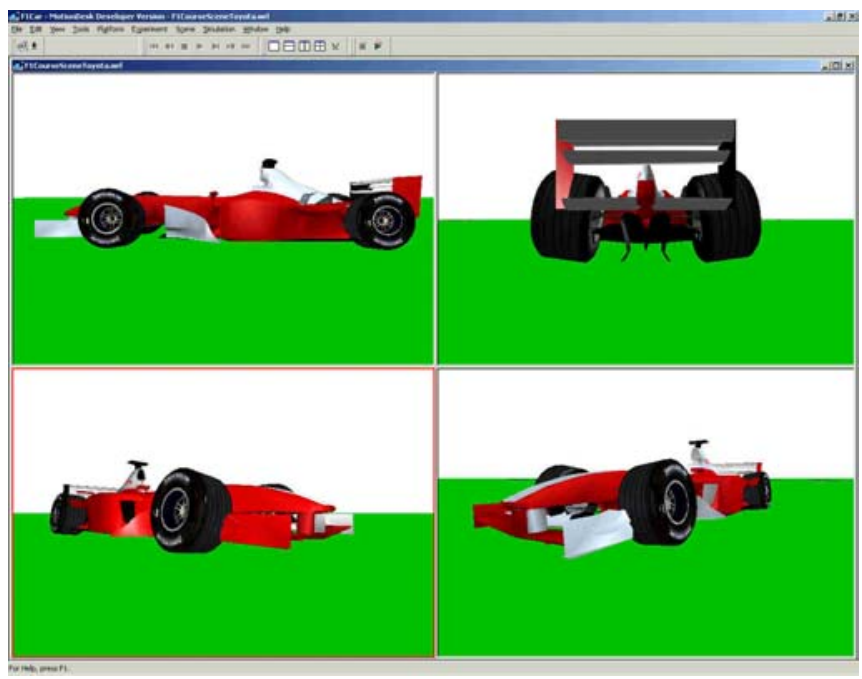

Figure 21: 3-D visualization of a racing vehicle

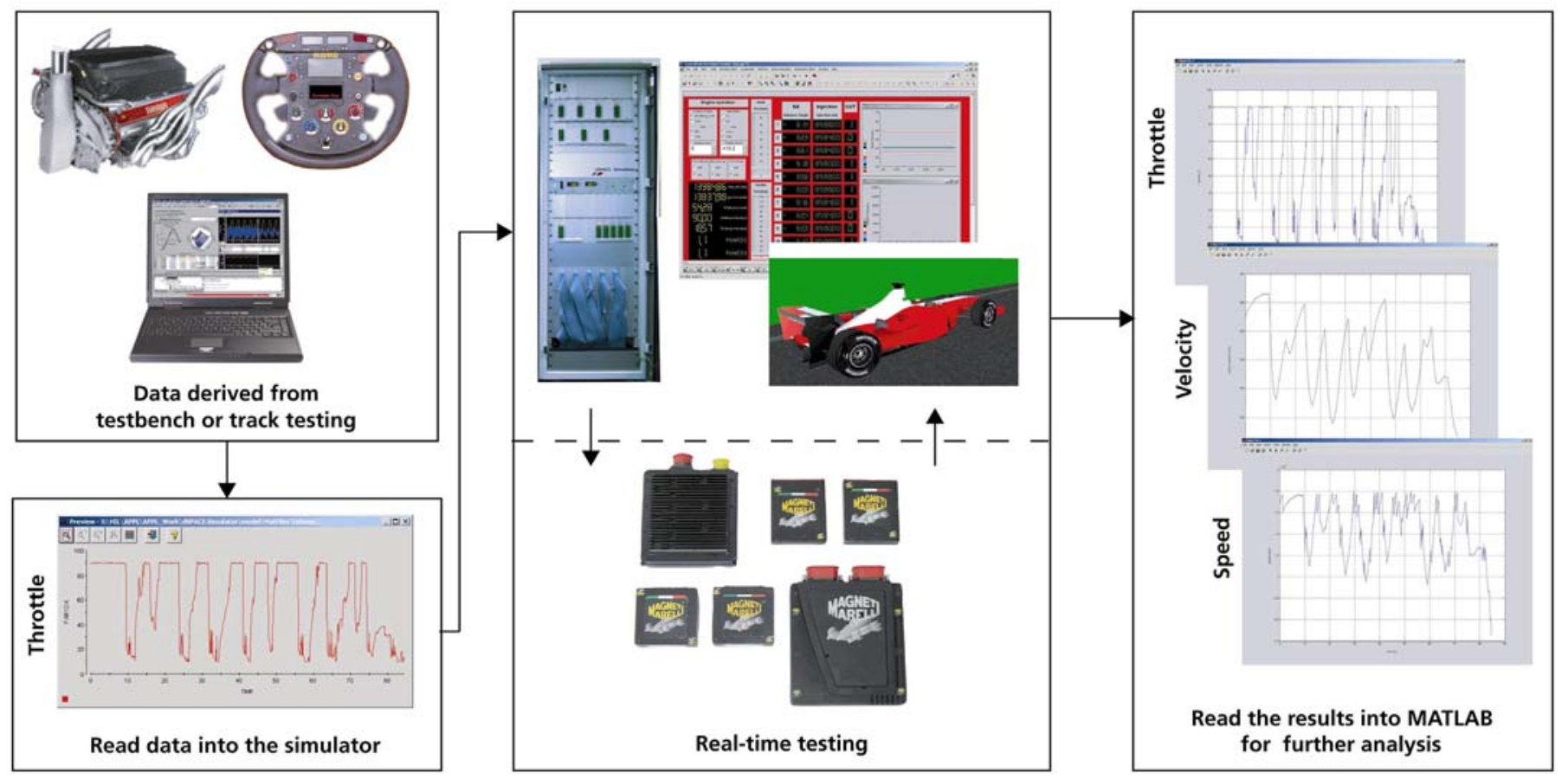

Figure 20: Sequence of an automated stimulus test using track data 


\section{TEST BENCH INTEGRATION}

In engine and powertrain development for motorsports, the test benches used are generally highly dynamic and allow transient processes during an acceleration phase (incl. gearshifts) and during start-up to be mapped sufficiently well. The load definitions for such test benches are based either on recorded track data or on predefined excitation scenarios, quasi in an open loop. However, to study the dynamic behavior of the powertrain, including the engine and the steering electronics, as they interact with the environment, the highly dynamic test bench has to be connected to the real-time simulation of the environment.

Some examples of this are optimizing traction control in F1 and rally vehicles, optimizing the gearshifts (up shift, down shift), and investigating the interaction between the engine and the transmission if one of them is not available. This can be the case, for example, with F1 teams that do not perform development at a single location, unlike Toyota and Ferrari.

A transmission test bench with a real gearbox and transmission ECU, a real or simulated engine controller, and electric motors applying loads to the drive shafts of the gearbox, is an example of this (Fig. 22). The behaviors of the engine and the vehicle are simulated in real time, and the resulting variables, drive shaft torque, and speed, are introduced via the electric drive and the electric brake.

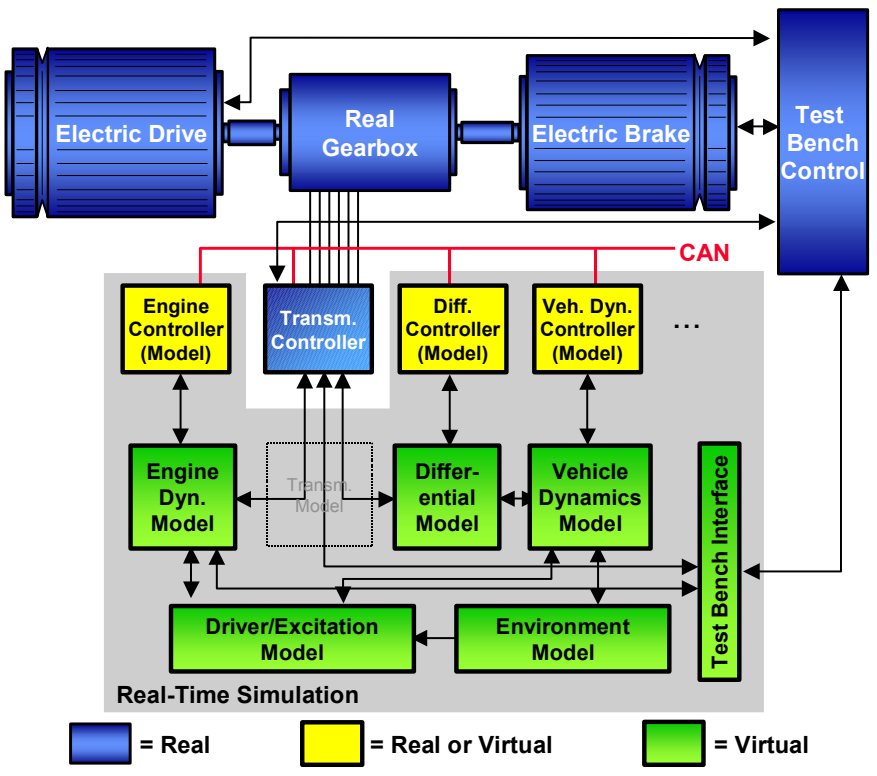

Figure 22: Test bench with real gearbox and transmission controller in combination with a real-time simulation (HIL) environment

Another example is shown in Fig. 23. Here the engine, transmission and differential are mounted on the test bench, while the vehicle dynamics, driver and the environment are simulated on an HIL system in real time. As in the first example above, the corresponding torque and speed signals of the drive shafts are simulated and applied to the powertrain by means of two electric brakes.

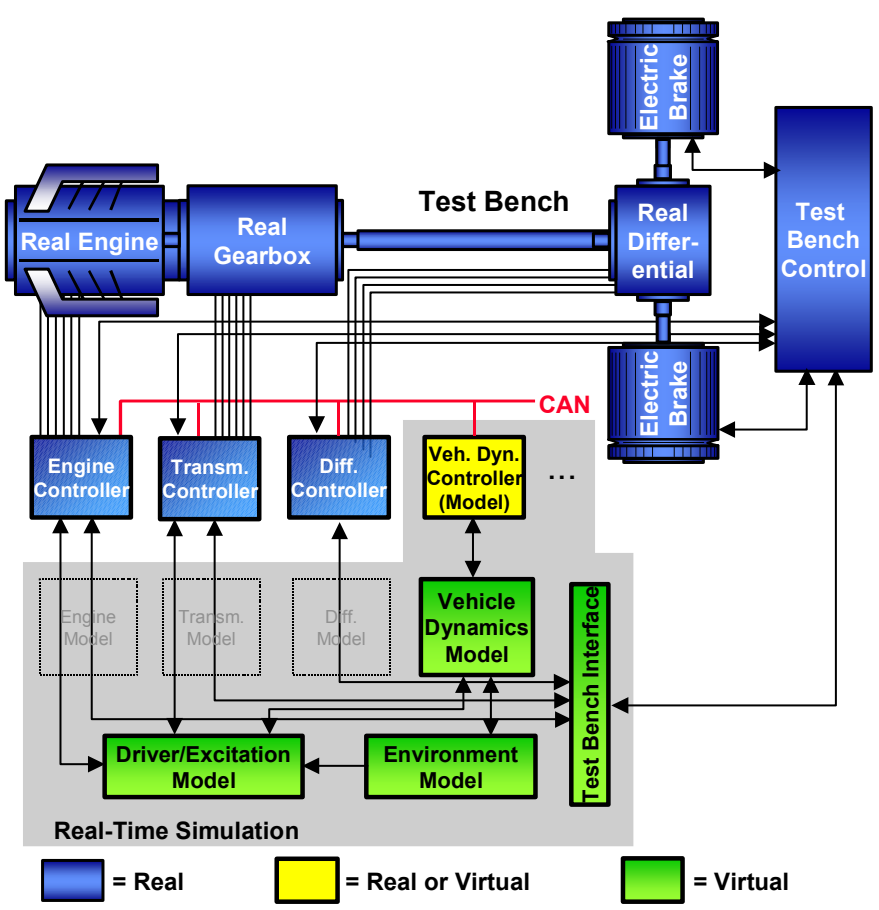

Figure 23: Test bench with real powertrain (engine, transmission, differential and corresponding controllers) in combination with a real-time simulation (HIL) environment

The test bench system and real-time simulation can be connected via CAN bus, Interbus, or Profibus, or via proprietary interfaces (such as a dSPACE AVL link). This test bench configuration has the following advantages:

- All ECUs can be integrated with real components at an early stage. This is particularly important if an ECU is available, but not the component to be controlled (engine, transmission, vehicle).

- The real testbed component does not need a dynamic model, nor do the real ECUs.

- Tests on the interaction between real and simulated components are highly reproducible.

- Suitable parameter sets for the ECUs can be generated even before the track test. This makes it likely that there will be fewer problems in transferring from the test bench to the racetrack, and greater software quality is achieved. 


\section{SUMMARY AND OUTLOOK}

This paper discusses the various options for using hardware-in-the-loop simulation in motorsports. Starting with the necessary hardware and software components of modern HIL test benches, concrete motorsports requirements and implemented solutions are presented. Finally, the integration of real-time simulation with concrete drive test benches and the resultant application scenarios are described. However, due to obligations of confidentiality, it was largely necessary to avoid detailed descriptions of concrete customer projects.

In the future, the authors see a clear trend towards a continued increase in the complexity of software and hardware systems in racing vehicles, as the basis for further performance growth in motorsports. The necessity of increased testing and more complex test systems will also rise in direct proportion to this. In both production and motorsports vehicles, determining variables that cannot be measured via substitute models in the ECUs will grow in importance. Observer systems such as those that are already in standard use for slip detection in vehicle dynamics control systems might also be used more in motorsports.

As active intervention is subject to tough regulations (especial in Formula One), the focus is mainly on existing actuators (especially the engine and transmission). This is different in rallying, where far more active intervention is allowed.

Future technical developments in Formula One remain difficult to predict, as frequent new proposals on the number of cylinders and displacement, and on the muchdiscussed "standard electronics", are made by the FIA. As regards the latter, it is still unclear whether only the hardware, or the software functions too, or even software parameterization, are to be standardized. It remains to be seen which way the discussion will go.

\section{REFERENCES}

[1] Wright, P.: Formula 1 Technology; SAE Society of Automotive Engineers, Warrendale, PA, USA, 2001.

[2] Urban, P.; Wältermann, P.; Henking, B.: Toyota Motorsport Racing Ahead with dSPACE (In German: Toyota Motorsport mit dSPACE auf der Überholspur. Automotive Engineering Partners 6/2001, S. 40. English Version available via: http://www.dspace.de/ ftp/papers/dspace AutEP 0111 e f22.pdf

[3] TESIS DYNAware: enDYNA®, veDYNA®, Product Description. Munich, Germany, http://www.tesis.de.

[4] Mechanical Simulation Corporation: CarSim ${ }^{\circledR}$ Product Description. Ann Arbor, Mi, USA, http://www.carsim.com.

[5] Hendricks, E. I Sorenson, S.: Mean Value Modelling of Spark Ignition Engines. SAE Paper No. 900616, Society of Automotive Engineers, Warrendale, PA, USA, 1990.

[6] Lamberg, K.; Richert, J.; Rasche, R.: A new Environment for Integrated Development and Management of ECU Tests. Society of Automotive Engineers, SAE Paper No. 2003-01-1024.

[7] Urban, P.: Verification of a decentralized ECU system for a Formula 1 car by means if Hardware-inthe-Loop-Simulation (in German). Conference: „Hardware-in-the-Loop-Simulation in Vehicle Development“, Haus der Technik, Essen, 2001.

[8] Plöger, M.; Sauer, J.; Büdenbender, M.; Held, J.; Costanzo, F.; de Manes, M.; di Mare, G.; Ferrara, F.; Montieri, A.: Testing Networked ECUs in a VirtualCar Environment. Society of Automotive Engineers, SAE Paper No. 2004-01-1724. 
Headquarters in Germany

dSPACE GmbH

Technologiepark 25

33100 Paderborn

Tel.: +49 5251 1638-0

Fax: +49525166529

info@dspace.de

www.dspace.de

\section{France}

dSPACE Sarl

Parc Burospace

Bâtiment 17

Route de la plaine de Gisy

91573 Bièvres Cedex

Tel.: +3316935 5060

Fax: +3316935 5061

info@dspace.fr

www.dspace.fr
USA and Canada

dSPACE Inc

28700 Cabot Drive . Suite 1100

Novi · Ml 48377

Tel.: +1 2485671300

Fax: +1 2485670130

info@dspaceinc.com

www.dspaceinc.com

United Kingdom

dSPACE Ltd.

2nd Floor Westminster House

Spitfire Close - Ermine Business Park

Huntingdon

Cambridgeshire PE29 6XY

Tel.: +44 1480410700

Fax: +44 1480410701

info@dspace.ltd.uk

www.dspace.ltd.uk 